\title{
Introduction to risk management plans in the Singapore context
}

Christine Su Ching $\underline{H o}^{1}$, BSc(Hons), Liesbet Li Bei $\underline{\operatorname{Tan}}^{1}$, BSc(Hons), Cheng Leng $\underline{\text { Chan }}{ }^{2}$, BSc(Hons)

ABSTRACT A risk management plan (RMP) contains an overview of important safety concerns associated with the use of a therapeutic product (TP) and proposed pharmacovigilance activities to monitor and manage these safety concerns in the post-approval stage. The Health Sciences Authority (HSA) of Singapore has legislated its requirements for RMPs as part of its product life cycle approach in the management of safety concerns associated with TPs. Under the Health Product (Therapeutic Products) Regulations enacted in November 2016, companies may be required to implement RMPs under the direction of HSA to help ensure that the benefits of TPs outweigh their risks. In this commentary, we present a case study on the local RMP for Dengvaxia ${ }^{\circledR}$, the world's first dengue vaccine, which has been approved for use in Singapore. We hope that this article raises awareness among healthcare professionals about the concept of RMPs and how RMPs are implemented locally.

Keywords: pharmacovigilance, risk management, risk management plans, risks

\section{INTRODUCTION}

Pharmacovigilance (PV) is defined as "the science and activities relating to the detection, assessment, understanding and prevention of adverse effects or any other drug-related problem ${ }^{\prime \prime} .^{(1)}$ It describes the process of ensuring that the safety profile of therapeutic products (TPs) on the local market remains optimal throughout the product life cycle (i.e. from pre-market to postmarket stages or until market withdrawal).

Historically, PV has been relatively reactive, with postmarket safety monitoring and signal detection relying mainly on spontaneous adverse event (AE) reporting. This system has worked well for some TPs but could lead to delayed signal detection for others, especially for TPs with rare AEs. Additionally, cases of high-profile safety-related market withdrawals such as that of cisapride (marketed in 1993, withdrawn in the United States [US] in 2000) and rofecoxib (marketed in 1999, withdrawn worldwide in 2004), indicated a need for more proactive PV in which preemptive post-market measures can be considered to minimise or mitigate serious safety concerns.

As a strategic approach towards more proactive PV, global regulations for risk management plan (RMP) requirements were introduced. Under RMP requirements, safety monitoring and risk minimisation processes are systematically planned and implemented by the companies. The first two international regulatory agencies to formalise the implementation of RMPs were the European Medicines Agency and the US Food and Drug Administration (FDA). In 2005, European Union legislation made it a requirement for companies to submit RMPs for all medicinal products during marketing authorisation applications. ${ }^{(2)}$ Subsequently, in 2007, US legislation authorised the US FDA to request for a Risk Evaluation and Mitigation Strategy at any point during a product's life cycle, if necessary, to ensure that the benefits of a drug outweigh the risks. ${ }^{(3)}$
In Singapore, the Health Sciences Authority (HSA) has legislated its requirements for RMPs as part of its product life cycle approach in the management of safety concerns associated with TPs. Under the Health Products (Therapeutic Products) Regulations enacted in November 2016, companies may be required to implement RMPs under the direction of HSA to help ensure that the benefits of TPs outweigh their risks. ${ }^{(4)}$

In this commentary, we present a case study on the local RMP implemented for Dengvaxia ${ }^{\circledR}$, the world's first dengue vaccine, to illustrate the RMP requirements directed by HSA in Singapore. We hope that this article will raise healthcare professionals' awareness of the concept of RMP and how they are implemented locally.

\section{WHAT IS AN RMP?}

An RMP is a detailed written plan prepared by pharmaceutical companies and submitted to the regulatory agencies, typically during the registration of TPs. It presents an overview of the important safety concerns associated with the TP and the proposed activities to be implemented to monitor and manage them in the post-approval phase. The RMP serves as an important tool for managing significant risks associated with a TP and may involve the implementation of risk minimisation activities beyond product labelling and routine AE reporting to ensure that the benefits of the product outweigh its risks. Effective implementation of the activities outlined in the RMP allows systematic tracking of the safety profile of TPs, which in turn facilitates prompt management of safety issues, leading to increased patient safety.

\section{WHEN IS AN RMP REQUIRED IN SINGAPORE?}

Currently, RMP submission is mandatory for all new chemical/ biological entities and biosimilars that seek product registration in Singapore. RMPs may also be requested for other TPs on a

${ }^{1}$ Product Risk Management Unit, Vigilance and Compliance Branch, ${ }^{2}$ Health Products Regulation Group, Health Sciences Authority, Singapore 
case-by-case basis, such as for TPs with new significant safety issues that are identified during the pre- or post-market stages. ${ }^{(5)}$

\section{MAIN COMPONENTS OF AN RMP SUBMITTED TO HSA \\ Safety concerns}

Important safety concerns associated with a TP may include:

- Important identified risk: where sufficient scientific evidence supports an association that is likely to impact the benefit-risk balance of the TP (e.g. adverse reactions confirmed in human studies that warrant strengthening of the package insert to include warnings and precautions/ contraindications) ${ }^{(6)}$

- Important potential risk: where scientific evidence signals suspicion of an association that could impact the benefitrisk balance of the TP, and close continual monitoring post-market is necessary to ascertain the association (e.g. toxicological findings in animal studies that have not been observed in human studies).

- Missing information: where there are gaps in knowledge related to safety for certain anticipated uses (e.g. long-term) or for use in particular patient populations of whom there is insufficient knowledge to determine whether the safety profile differs from those characterised so far.

The RMP is a dynamic document that should be updated throughout the life cycle of the TP as knowledge about the TP accumulates. For example, a safety concern that is initially categorised as an 'important potential risk' may be reclassified as an 'important identified risk' or removed from the RMP when new scientific or clinical data associated with the TP becomes available.

\section{Proposed pharmacovigilance activities}

Proposed PV activities help to further elucidate and/or to minimise safety concerns. They can be categorised into routine activities, which are standard measures applied to all TPs, and additional activities, which are applied to selected TPs with safety concerns that cannot be adequately addressed via routine monitoring activities (e.g. teratogenicity or potentially life-threatening adverse reactions). Over time, these additional activities are reviewed on their effectiveness in managing the safety concerns, and modification or discontinuation of these activities may be proposed. Examples of routine and additional activities to monitor (Box 1) and minimise (Box 2) safety concerns are shown.

We present a case study to illustrate the components of a local RMP that was put in place and is continually reviewed to ensure that the benefit-risk profile of Dengvaxia remains positive throughout its product life cycle.

\section{CASE STUDY}

Local RMP for Dengvaxia (chimeric yellow fever dengue, dengue virus serotypes 1-4)

Dengvaxia, the world's first vaccine for dengue prevention, was approved in Singapore in October 2016. It is indicated for the prevention of dengue disease caused by four strains of dengue
Box 1. Examples of routine and additional activities to monitor safety concerns:

\section{Routine}

- Timely reporting of local serious adverse events

- Timely update on significant safety issues that may influence the overall benefit-risk profile of a therapeutic product (TP)

- Timely update on safety-related regulatory actions taken by other regulatory agencies

- Preparation of periodic benefit-risk evaluation reports (PBRERs)*; submission required for selected TPs

\section{Additional}

- Implementation of active surveillance programmes

- Conducting post-marketing safety studies (e.g. including longterm follow-up of patients enrolled in clinical trials)

- Conducting additional clinical studies (e.g. pharmacokinetic studies to determine if there is a higher risk of adverse events in a certain group of patients)

*A PBRER is a report evaluating a product's benefit-risk profile, produced by the pharmaceutical company at six-monthly intervals or annually.

Box 2. Examples of routine and additional activities to minimise
safety concerns:
Routine
- Safety updates to the package insert
Additional
- Development of educational materials for physicians and/or
patients
- Issuance of DHCPLs (Dear Healthcare Professional Letters)
- Implementation of restricted access programme (e.g. supply to
- Imlected physicians/specialists)

virus (DENV-1, DENV-2, DENV-3 and DENV-4) in individuals aged $12-45$ years. At the point of product approval, based on the available clinical data, a higher minimum recommended age of 12 years was set (instead of nine years) due to concerns that young children could have a higher risk of severe dengue following vaccination, as well as inconsistent risk reduction in vaccinated children aged 9-11 years.

In addition, clinical studies submitted for registration showed that Dengvaxia was more effective in individuals with previous dengue infection. There was also a theoretical concern that vaccination with Dengvaxia in individuals who were dengue-naive could increase the risk of severe dengue should they subsequently become infected with dengue. ${ }^{(7)}$ Hence, the local package insert of Dengvaxia provided recommendations to consider serostatus testing, if available, in individuals with an unknown history of prior dengue exposure, to determine past infection status and inform the benefit-risk considerations following vaccination with Dengvaxia. These recommendations were conveyed to medical practitioners by both HSA and the Ministry of Health $(\mathrm{MOH})$ upon the approval of the vaccine. At that time, Singapore was the only country that required these recommendations for Dengvaxia. $\mathrm{MOH}$ subsequently worked with the Singapore General Hospital and National University 
Hospital to make the serology test available to medical practitioners.

Under the direction of HSA, a local RMP with additional activities to monitor and minimise the aforementioned concerns was implemented by the company at the point of product approval. This was to ensure that safety concerns identified were monitored and managed such that the benefit-risk profile of Dengvaxia remained positive throughout the product's life cycle. The local Dengvaxia RMP included the following:

- Mandatory reporting to HSA by the company of all local spontaneous, serious AEs associated with Dengvaxia no later than 15 days after becoming aware of the event. These may include cases of dengue infections following vaccination, with or without hospitalisation.

- Submission of significant overseas AEs associated with Dengvaxia to HSA by the company (including dengue cases with or without hospitalisation).

- $\quad$ Provision of regular safety and efficacy/effectiveness updates regarding Dengvaxia through the submission of periodic benefit-risk evaluation reports (PBRERs).

- $\quad$ Timely notifications to HSA on safety-related regulatory actions taken by other regulatory agencies regarding Dengvaxia.

- Development of educational materials, namely physician educational material and patient medication guide.

- Submission of monthly local sales volume data to HSA to facilitate understanding of local product usage.

The components of the RMP, which include company notifications of local and international AEs as well as PBRER submission, are complementary to ensure timely detection of important safety signals and efficacy issues so that prompt regulatory interventions can be taken, if required.

The PBRER provides periodic analyses of the safety, efficacy and effectiveness of Dengvaxia over its product life cycle, based on relevant new information and cumulative knowledge obtained from various data sources. These sources included, but were not limited to, global AE reports, ongoing or completed clinical studies, post-authorisation safety studies (long-term follow-up of patients to provide greater insight into the safety profile) and published literature. Through these measures, cases of dengue following vaccination with or without hospitalisation were closely monitored.

In addition to risk management activities carried out by the company, healthcare professionals were encouraged to report AEs following the use of Dengvaxia directly to HSA and/or to the company to contribute to safety monitoring in Singapore. Additionally, healthcare professionals were also advised to obtain the history of dengue vaccination in patients diagnosed with dengue. This information, when included in the dengue notification to $\mathrm{MOH}$, would greatly facilitate HSA's ongoing monitoring of the safety and efficacy of Dengvaxia.

Apart from safety monitoring, the local Dengvaxia RMP also aimed to strengthen communication of key safety information to healthcare professionals. Hence, educational materials detailing the approved indications and limitations of Dengvaxia, particularly for individuals without previous dengue infection, were developed by the company and submitted to HSA for review and approval prior to distribution to healthcare professionals. In these materials, pertinent information on specific safety concerns for doctors and patients to look out for, as well as recommendations for appropriate measures to minimise or address these concerns following vaccination, were provided. The HSA-approved educational materials also carried the statement "This document has been approved by HSA on [Date]" to aid healthcare professionals in differentiating between educational materials requested by HSA and company-initiated promotional materials. Healthcare professionals were encouraged to utilise these educational resources to support them in making informed decisions for their patients and as an aid in counselling their patients.

\section{Review and update of local Dengvaxia RMP}

The local RMP implemented by the company is continually reviewed and updated when new safety information emerges. Following marketing approval for Dengvaxia in Singapore, findings from a post-marketing exploratory study conducted by the company to assess the long-term safety and efficacy of Dengvaxia became available. This study confirmed the earlier postulated increased risk of clinically severe dengue following vaccination with Dengvaxia in dengue-naive individuals. As this important finding affected the vaccine's benefit-risk profile in dengue-naive patients, a Dear Healthcare Professional Letter was issued by the company, in consultation with HSA, to heighten communication about the need to conduct serostatus testing in individuals whose serostatus is unknown, as well as the updated recommendations that vaccination with Dengvaxia should not be recommended to dengue-naive and seronegative individuals. ${ }^{(8)} \mathrm{HSA}$ also issued updates on its website $^{(9,10)}$ to reinforce the communication of this new finding to the public. It subsequently worked with the company to strengthen the package insert and educational materials to reflect the new warnings and advisories.

\section{CONCLUSION}

An RMP serves as an important tool to highlight safety concerns identified for the TP during the pre-market phase so that appropriate activities can be proposed and implemented in the post-market phase to adequately monitor and manage them. Our case study on Dengvaxia illustrated the components of a local RMP that was implemented for TPs in Singapore under the direction of HSA and how it was subsequently updated following the emergence of new findings. We hope this commentary will raise healthcare professionals' awareness of the concept of RMPs and how they are applied locally.

\section{ACKNOWLEDGEMENTS}

We thank Dr Dorothy Toh (Assistant Group Director, Vigilance, Compliance and Enforcement Cluster, Health Products Regulation Group, HSA), Ms Jalene Poh (Director, Vigilance and Compliance Branch, HSA) and Ms Adena Lim (Deputy Director, Vigilance and Compliance Branch, HSA) for their support and guidance for this 
manuscript; and Dr Anuradha Poonepalli (Regulatory Consultant, Therapeutic Products Branch, HSA), Ms Sally Soh (Regulatory Consultant, Vigilance and Compliance Branch, HSA) and Ms Peck Li Fung (Senior Regulatory Specialist, Vigilance and Compliance Branch, HSA) for their advice regarding the manuscript.

\section{REFERENCES}

1. World Health Organization. Essential medicines and health products: pharmacovigilance. Available at: http://www.who.int/medicines/areas/ quality_safety/safety_efficacy/pharmvigi/en/. Accessed August 15, 2019.

2. European Medicines Agency, Committee for Medicinal Products for Human Use, EMEA/CHMP/96268/2005 Guideline on risk management systems for medicinal products for human use, London: EMEA, 2005.

3. US Food and Drug Administration. Approved Risk Evaluation and Mitigation Strategies (REMS). Available at: https://www.accessdata.fda.gov/scripts/cder/ rems/index.cfm. Accessed August 15, 2019.

4. Singapore Statutes Online. Health Products Act (Chapter 122D). Health Products (Therapeutic Products) Regulations 2016. Available at: https://sso.agc.gov.sg/ SL/HPA2007-S329-2016? DocDate=20180301. Accessed August 15, 2019.

5. Health Sciences Authority, Singapore. Guidance for Industry and Applicants. 1. Post-Marketing Vigilance Requirements for Therapeutic Products (November 2016). Available at: https://www.hsa.gov.sg/content/hsa/ en/Health_Products_Regulation/Safety_Information_and_Product_Recalls/
Report_Adverse_Events_related_to_health_products/Guidance_for_Industry_ and_Applicants.html. Accessed August 29, 2019.

6. European Medicines Agency. Guideline on good pharmacovigilance practices (GVP). Module V - Risk Management systems (Rev 2). 28 March 2017. Available at: https://www.ema.europa.eu/documents/scientific-guideline/guideline-goodpharmacovigilance-practices-module-v-risk-management-systems-rev-2_en.pdf. Accessed August 15, 2019.

7. Health Sciences Authority, Singapore. HSA Approves Dengvaxia Vaccine. Available at: http://www.hsa.gov.sg/content/hsa/en/News_Events/ HSA_Updates/2016/hsa-approves-dengvaxiavaccine.html. Accessed August 15, 2019.

8. Health Sciences Authority, Singapore. Dear Healthcare Professional Letter: Important findings from a supplementary exploratory study on Dengvaxia ${ }^{\circledR}$ to assess long term safety and efficacy of Dengvaxia ${ }^{\circ}$ from Phase IIb/III studies. Available at: https://www.hsa.gov.sg/content/hsa/en/Health_Products_ Regulation/Safety_Information_and_Product_Recalls/Dear_Healthcare_ Professional_Letters/2017/important-findingsfromasupplementaryexploratorys tudyondengvaxiat.html. Accessed August 15, 2019.

9. Health Sciences Authority, Singapore. HSA Updates on Additional Risk of Dengvaxia ${ }^{\circledR}$ in Individuals with No Prior Dengue Experience. Available at: http://www.hsa.gov.sg/content/hsa/en/News_Events/HSA_Updates/2017/ dengvaxiaupdate.html. Accessed August 15, 2019.

10. Health Sciences Authority, Singapore. HSA Further Updates on Dengvaxia $®$. Available at: http://www.hsa.gov.sg/content/hsa/en/News_Events/HSA_ Updates/2017/dengvaxiafurtherupdates.html. Accessed August 15, 2019. 